\title{
Special Convolutional Neural Network for Identification and Positioning of Interstitial Lung Disease Patterns in Computed Tomography Images
}

\author{
Sunita Agarwala ${ }^{a, *}$, Abhishek Kumar ${ }^{b, * *}$, Ashis Kumar Dhara ${ }^{c, * * *}$, Sumitra Basu Thakur ${ }^{d * * * *}$, \\ Anup Sadhu ${ }^{e, * * * * *}$, and Debashis Nandi ${ }^{a, * * * * * *}$ \\ ${ }^{a}$ Computer Science and Engineering National Institute of Technology, Durgapur, 713209 India \\ ${ }^{b}$ School of Computer and Information Sciences University of Hyderabad, Hyderabad, 500046 India \\ ${ }^{c}$ Electrical Engineering National Institute of Technology, Durgapur, 713209 India \\ ${ }^{d}$ Department of Chest and Respiratory Care Medicine, Medical College, Kolkata, 700073 India \\ ${ }^{e}$ EKO Diagnostic, Medical College, Kolkata, 700073 India \\ *e-mail: sunitacse10@gmail.com \\ **e-mail: abhishekcse10@gmail.com \\ ***e-mail: dear.ashis79@gmail.com \\ ****e-mail: drsbthakur@yahoo.co.in \\ *****e-mail:sadhujee@gmail.com \\ ******e-mail:debashis@cse.nitdgp.ac.in
}

\begin{abstract}
In this paper, automated detection of interstitial lung disease patterns in high resolution computed tomography images is achieved by developing a faster region-based convolutional network based detector with GoogLeNet as a backbone. GoogLeNet is simplified by removing few inception models and used as the backbone of the detector network. The proposed framework is developed to detect several interstitial lung disease patterns without doing lung field segmentation. The proposed method is able to detect the five most prevalent interstitial lung disease patterns: fibrosis, emphysema, consolidation, micronodules and groundglass opacity, as well as normal. Five-fold cross-validation has been used to avoid bias and reduce over-fitting. The proposed framework performance is measured in terms of F-score on the publicly available MedGIFT database. It outperforms state-of-the-art techniques. The detection is performed at slice level and could be used for screening and differential diagnosis of interstitial lung disease patterns using high resolution computed tomography images.
\end{abstract}

Keywords: Convolutional Neural Networks, detection of interstitial lung disease patterns, high-resolution computed tomography, faster region-based convolutional network based object detection and F-score

DOI: $10.1134 / \mathrm{S} 1054661821040027$

\section{INTRODUCTION}

A group of nearly 200 different pulmonary diseases is combined together to form a common group known as interstitial lung disease (ILD) [32]. The radio-histopathological finding of the majority of diseases from this group suggests the progressive scarring of lung tissues, which would cause increased breathlessness in the patient. It also disturbs the flow of oxygen into the bloodstream. The group shows similar clinical properties among them. It makes difficult to distinguish among them. It makes higher intra and inter variability of the observers. Even experienced medical experts find it difficult for differential diagnoses. Earlier diagnosis improves the

Received November 23, 2020; revised May 24, 2021; accepted July 4, 2021 chance of cure and recovery with less time. High-resolution computed tomography (HRCT) images are identified as ideal imaging tools for studying patterns of ILD as it provides higher resolution as compared to CT images. The most prevalent ILD patterns can be broadly classified into five major groups: fibrosis, emphysema, consolidation, micronodules, groundglass opacity (GGO), and normal. All these patterns are shown in Fig. 1.

Different imaging modalities exist in the medical domain, such as X-ray, CT, magnetic resonance imaging (MRI), positron emission tomography (PET). Image analysis plays an essential role in the identification of different abnormalities. Hence, intelligence is applied to analysis imaging technique that reduced human intervention to make clinical and research decisions in the medical domain [19]. The deep learning approach recently shows an impressive perfor- 
(a)

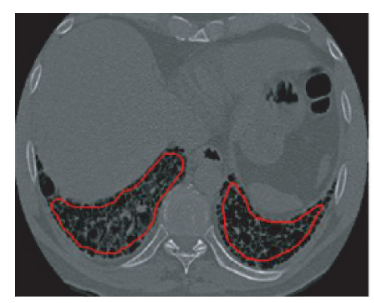

(b)

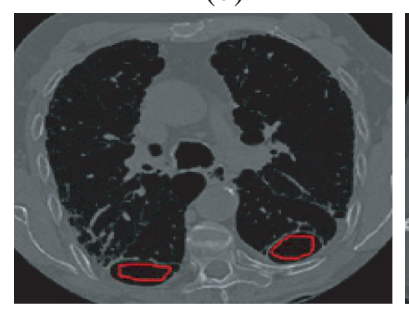

(c)

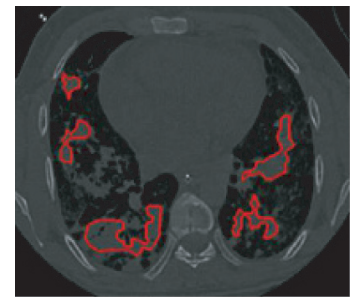

(d)

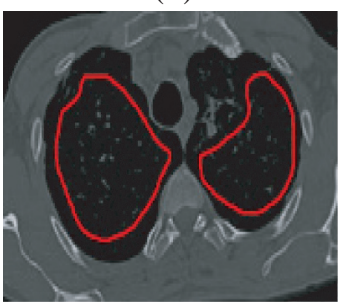

(e)

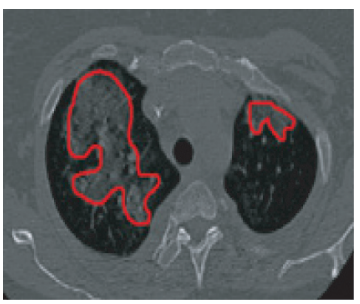

(f)

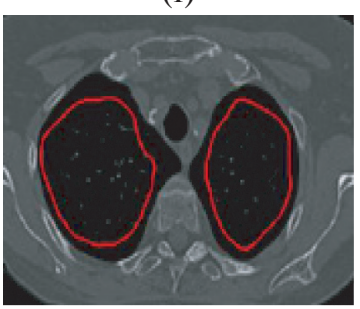

Fig. 1. Different ILD patterns are: (a) fibrosis, (b) emphysema, (c) consolidation, (d) micronodule, (e) GGO, and (f) normal lung.

mance in different fields: object detection, classification, and segmentation. Sarvamangala et al. [23] have explained the importance of CNN for analyzing the images. The primary advantage of CNN architecture is extracting features from an image without human intervention. This is the main reason researchers have applied CNN based techniques in the different medical domains like detection of ILD patterns [1, 16], classification of ILD [22, 30], lung cancer classification [20], segmentation, prediction, and staging of COVID-19 [4, 31].

Bermejo-Peláez et al. [22] have used a deep learning approach for the classification of ILD patterns. They have developed a CNN architecture that comprises three different architectures such as 2D, 2.5D, and $3 \mathrm{D}$ for the classification of ILD abnormalities. To calculate the network's performance, they have extracted radiographic tissue of 37424 from 208 CT images for eight classes. The result is reported in terms of sensitivity and specificity, i.e., 91.41 and $98.18 \%$, respectively. Detection of ILD patterns is a significant prevalence to measure the severity of COVID-19 in this pandemic situation. Chassagnonn et al. [4] proposed a method for finding the stage of patients affected by COVID-19 pneumonia. The authors have compared three network architectures as: CovidENet, CovidE2D, and CovidE3D. The results are evaluated in terms of dice coefficient and dice Hausdorff distance. The CovidENet has been given the best result. The mean dice coefficient and Hausdorff distance are 0.70 and 8.9, respectively, for CovidENet. Wang et al. [31] have used the hybrid encoder technique for better segmentation of lesion features for COVID-19. Those features are help to distinguish between general lung lesions with infected lung lesions. The performance is reported in
NSD, mean DSC, F1-score, MCC, sensitivity, and accuracy with the value of $0.704,0.735,0.682,0.707$, 0.994 , and 0.716, respectively. Therefore, in this paper, we have applied a deep learning approach for the identification of ILD patterns. The state-of-theart works on detecting ILD patterns are broadly divided into two categories: patch-based methods and slice-based methods.

\subsection{Patch-Based Classification}

Most of the reported works concentrated on the patch-based classification of ILD patterns, where patches are extracted manually by expert radiologists [5, 7, 26, 33]. Informative features are extracted from several ILD patches with the help of different feature extraction techniques for the classification of ILD patterns. They have been used in [10] to produce multiscale filters and the generated responses were saved as corresponding features. The selection of an appropriate classifier is very important. A plethora of techniques and methods can be found in the literature for this topic. Some of the methods of prominence are $k$-nearest neighbors $[9,15,18,21]$, artificial neural network [14], and support vector machines [3, 5, 14, 21, 28, 29, 34, 35]. Depeursinge et al. [5] have developed a near-affine-invariant texture-based feature descriptor based on wavelet transformation. This method was evaluated for the classification of five HRCT patterns (healthy, emphysema, GGO, fibrosis, and micronodules) and achieved a classification accuracy of $76.9 \%$. Song et al. [26] used texture and gradient features for patch-based classification of five HRCT patterns, as mentioned earlier. Rotation-invariant Gabor-local binary patterns texture descriptor and gradient 
descriptor of multicoordinate histogram of oriented gradients have been used to represent HRCT patterns. The accuracy of classification using support vector machine reported in terms of F-score for each pattern. F-scores for healthy, emphysema, GGO, fibrosis, and micronodules are 84, 75.3, 78.2, 84.1, and $85.7 \%$. To handle intraclass variation and interclass ambiguity in HRCT patterns, Song et al. [27] developed a large margin local estimate classification model and improved classification accuracy. The majority of these techniques have used handcrafted features, and the method fails for a new data or pattern which is not adaptive. Learning features are extracted using the restricted Boltzmann machine (RBM) to cope up with the above issue. RBM is a generative stochastic artificial neural network that can be trained either in unsupervised or supervised ways.

Huang et al. [14] have compared two different network architectures for the classification of ILD patterns. One is based on deep CNN architecture that comprises six different convolutional layers followed by batch normalization layers and ends with a fully connected layer. The network takes input as patches of size $32 \times 32$ that is extracted from ILD images. Each layer having a kernel size of $2 \times 2$, and the number of kernels adds in increasing order from 32 up to 192. In this architecture, ReLU is used as an activation function. Another architecture is based on a deep convolution autoencoder (DCAE). To train, these network architecture, authors have been used ADAM optimizer and cross-entropy loss function. The learning rate of 0.0008 is used, which is decreased by $10 \%$ after every 20 epochs. The main contribution of authors they have been used twostage transfer learning. The results are evaluated in terms of F1-score. The authors achieved the best results using two-stage transfer learning in both network architectures. The F1-scores are 0.9791 and 0.9810 for CNN and DCAE, respectively. The limitation of this method used patch-based instead of slice-based input images.

Deep learning based image processing techniques using convolutional neural networks (CNNs) have registered their strong presence and effectiveness while dealing with natural color images. Anthimopoulos et al. [2] have developed a CNN for the classification of ILD patterns in HRCT images. LeakyReLU was used as an activation function. The cross-entropy loss is used to optimize ADAM. The network is evaluated for seven HRCT patterns: healthy, micronodules, GGO, consolidation, honeycombing, reticulation, and combination of $\mathrm{GGO} /$ reticulation, and achieved a classification accuracy of $85 \%$. The shallow design of this network restricts it from utilizing the adequate advantage from the ability of deep CNN layers. However, patch-based classification is clinically less desirable as it cannot be used for screening of HRCT patterns at slice level.

\subsection{Slice-Based Classification}

Gao et al. [11] used pretrained AlexNet to classify HRCT slices depending on the presence of pathology. Input images are rescaled to fit AlexNet architectural design, as to exploit the advantages of colored images, they have used different Hounsfield unit (HU) windows to artificially create the three channels. The accuracy of classification is better for emphysema, but the poor classification is observed for micronodules and healthy patterns due to change of contrast. Shin et al. [24] improved the slice level classification accuracy of HRCT slices using GoogLeNet. The main objective of the slice test is to classify the slice based on the presence of five ILD patterns. Anthimopoulos et al. [1] have proposed a CNN network with dilated filters to segmentation ILD patterns. This network input images are any arbitrary size of lung CT and outputs generated by this network are label maps. This network consists of eight convolutional layers having different dilation rates that increase exponentially. This helps to increase the receptive field and also linearly grown number of parameters. The result is evaluated on 172 HRCT slices collected from two hospitals such as Geneva University Hospital and Bern University Hospital. This network achieves an accuracy of $81.8 \%$. The network was trained by minimizing the categorical cross-entropy using the ADAM optimizer with a learning rate of 0.0001 .

\subsection{Contribution}

Localization of ILD patterns in HRCT image slices is clinically more desirable than patch level classification for screening of ILD. The present work focuses on the localization of several ILD patterns in a HRCT slice using a faster region-based convolutional network ( $\mathrm{R}-\mathrm{CNN})$ based object detection network. GoogLeNet architecture is modified for reduction of complexity and used as a feature extractor of faster R-CNN object detector in the proposed framework. Feature map is obtained from GoogLeNet and fed to region proposal network (RPN) to serve as detection network. The number of the bounding box enclosing ILD patterns in a slice would help in pathology quantification. This framework is fast, accurate, and could be used in the screening of ILD using HRCT image slices. The proposed work reveals that the deep learning based techniques could eliminate the lung field extraction step for the identification of ILD patterns.

\section{METHODS}

\subsection{Database}

Publicly available MedGIFT database [8] is used for this study. The database has 108 annotated image series of HRCT. It consists of 17 different patterns of ILD with sizes $512 \times 512$. Total 1946 ROIs were provided from 
Table 1. Total number of image slice with respect to different type of patterns and there corresponding total number of region of interest (ROI) present for that specific patterns are acquired from MedGIFT database

\begin{tabular}{l|c|c}
\hline ILD patterns & $\begin{array}{c}\text { Total number } \\
\text { of image slice }\end{array}$ & $\begin{array}{c}\text { Total number } \\
\text { of ROIs }\end{array}$ \\
\hline Consolidation & 116 & 194 \\
Emphysema & 71 & 108 \\
Fibrosis & 293 & 479 \\
GGO & 241 & 415 \\
Healthy & 69 & 98 \\
Micronodule & 154 & 277 \\
Total & 944 & 1571 \\
\hline
\end{tabular}

Table 2. Average number of images used in each pattern for training and testing purpose

\begin{tabular}{l|c|c|c}
\hline ILD patterns & $\begin{array}{c}\text { Training } \\
\text { images before } \\
\text { augmentation }\end{array}$ & $\begin{array}{c}\text { Training } \\
\text { data after } \\
\text { augmentation }\end{array}$ & $\begin{array}{c}\text { Testing } \\
\text { images }\end{array}$ \\
\hline Consolidation & 93 & 557 & 23 \\
Emphysema & 57 & 341 & 14 \\
Fibrosis & 234 & 1406 & 59 \\
GGO & 193 & 1157 & 48 \\
Healthy & 55 & 331 & 14 \\
Micronodule & 123 & 739 & 31 \\
\hline
\end{tabular}

108 image series of HRCT. In this work, the five most prevalent ILD patterns and healthy patterns are considered. The database is multipattern in nature, which means there is a probability of more than one pattern in the same slice. Statistical detail has been provided in Table 1 for an aforementioned pattern.

\subsection{State-of-the-Art CNN Architectures}

Several networks like VGGNet, GoogLeNet, and ResNet have been used for the natural image classification task. VGGNet [25] uses only $3 \times 3$ convolutional layers, those are stacked on top of each other. The networks have varying depths ranging from 11 to 19. The architectural design of GoogLeNet [6] is different as the depth of the network is increased vertically as well as horizontally with the help of inception modules. This was the naive idea of an inception module, where three convolution networks with filter size $1 \times 1,3 \times 3$, and $5 \times 5$ are used in parallel together with a $3 \times 3$ max-pooling layer. In this network, a
$1 \times 1$ convolutional layer is introduced before the $3 \times 3$ and $5 \times 5$ layers. The $1 \times 1$ layer acts as an instrument for the reduction of computational complexity. The final module comprises network in the network layer, large and medium size convolution filter, and pooling operation. This network layer is capable of extracting information at a fine level. The $5 \times 5$ filter provides a wider receptive field, and the pooling operation reduces the spatial sizes and also looks after overfitting. ResNets [13] are the deepest networks with a maximum of 152 layers. The deeper networks face a degradation problem when they start to converge. In this network concept of residual learning is introduced to address the degradation problem. The residual block is an ensemble of conv-relu-conv series.

\subsection{Faster R-CNN Based Object Detector with GoogLeNet as Backbone}

In the proposed framework (Fig. 2), GoogLeNet architecture with a reduced inception module is used to extract features for finding region proposals. Instead of nine, five inception modules are used in GoogLeNet in order to reduce network parameters. The fifth inception block provides features, which is used for finding region proposal. The region proposal network (RPN) has two outputs such as RPN classification head and RPN regression head. The RPN classification output layer predicts an anchor box that is an object or background. The RPN regression output layer predicts box offsets (left corner coordinates, width, and height). The feature map is also connected to ROI max-pooling layer to pool features for classifying the pooled regions. Classification and bounding box regression head are attached with the ROI pooling layer. After detection of region proposals for an object, the class score is calculated for every region. The proposed network is trained from scratch with a learning rate of 0.00001 . The training was performed up to 35 epochs. ADAM [17] issued to optimize the crossentropy based loss function.

\section{EXPERIMENTAL RESULTS}

\subsection{Dataset for Training and Testing}

Table 1 shows that the average number of training and testing images for each pattern is very less. Unlike classical techniques, where the feature is manually defined and calculated, in deep learning, the feature is extracted with the help of a training image. The true potential of CNNs can only be realized if the training data is large enough to effectively train the network so that it captures all the relevant features. There is always a shortage of annotated quality data in the medical domain. Therefore, data augmentation techniques (flip, rotation, change of contrast, and addition of Gaussian noise) have been used to increase the available data. With an increased number of existing training data, the chances of overfitting also get reduced. 


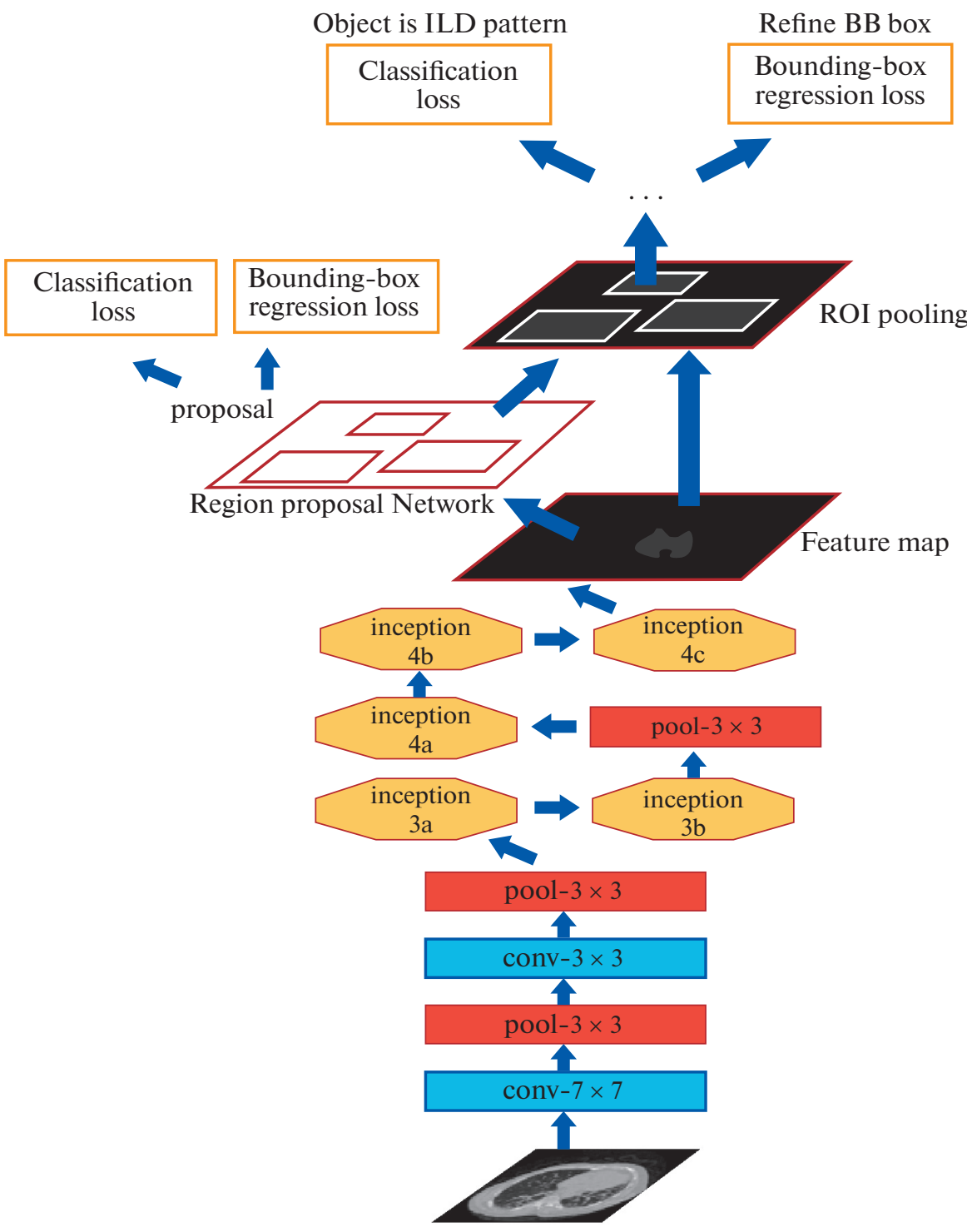

Fig. 2. Faster R-CNN based object detector.

The number of testing and training images for all ILD patterns after applying data augmentation techniques is shown in Table 2.

\subsection{Quantitative Results of Slice Test}

The proposed framework performance is compared with Shin et al. [24] method in terms of Fscore. It assigns a score based on precision and recall values [12]. Precision is the ratio of true-positive detection to all detections, whereas recall is the ratio of true-positive detection to ground-truth instances. An overlap greater than 0.1 between the ground truth bounding box and the object's detected bounding box is considered true positive. F-score is simply the harmonic mean of precision and recall. The value of the F-score varies between $(0,1)$, where 1 is the best case and 0 is the worst case. Here, $\beta$ is a positive real factor, while putting $\beta=1$ in Eq. (3), we got $F_{1}$-score

$$
\begin{gathered}
\text { Precision }=\frac{x_{\mathrm{tp}}}{x_{\mathrm{tp}}+x_{\mathrm{fp}}}, \\
\text { Recall }=\frac{x_{\mathrm{tp}}}{x_{\mathrm{tp}}+x_{\mathrm{fn}}}, \\
=\left(1+\beta^{2}\right)\left(\frac{F_{\beta}-\text { Score }}{(\text { Precision.Recall }}\right), \\
F_{1} \text {-Score }=2\left(\frac{\text { Precision.Recall } \left.^{2}\right)+ \text { Recall }}{\text { Precision }+ \text { Recall }}\right),
\end{gathered}
$$


(a)

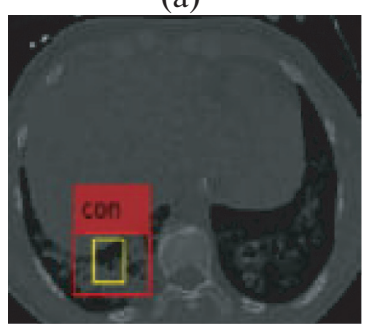

(d)

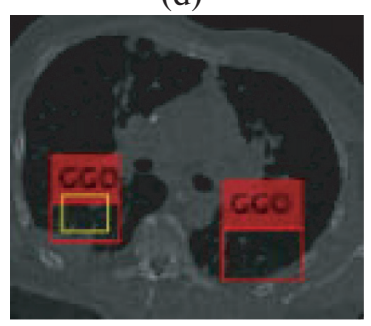

(b)

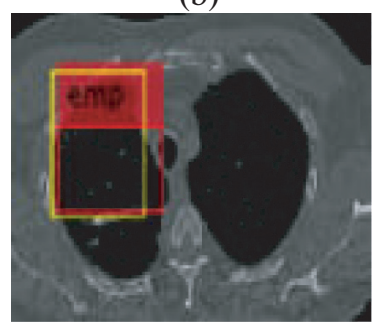

(e)

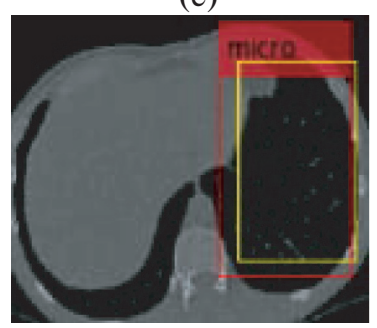

(c)

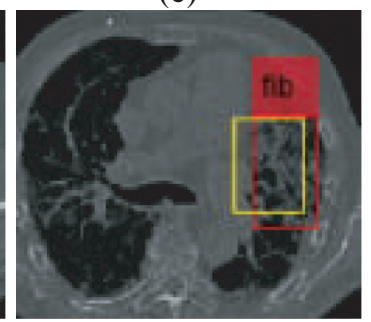

(f)

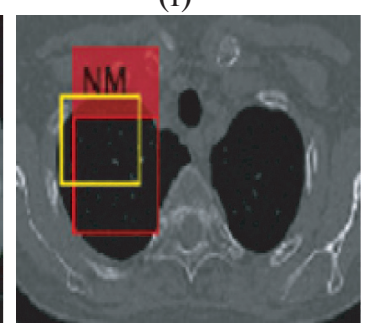

Fig. 3 Results of detection of the proposed framework for (a) consolidation (b) GGO, (c) emphysema, (d) micronodules, (e) fibrosis, and (f) normal lung field, respectively. The ground truth and output bounding box is represented by red and yellow color respectively.

where $x_{\mathrm{tp}}, x_{\mathrm{fn}}$, and $x_{\mathrm{fp}}$ are number of true positives, false negative, and false positives, respectively.

The performance of the proposed framework is calculated in terms of F-score (Eq. (4)) using five-fold crossvalidation. Table 3 shows the comparison of the F-score of the proposed framework with Shin et al. [24]. The proposed method outperforms the competing techniques for all patterns except fibrosis. In the MedGIFT database, all the ROIs with particular pathology in HRCT slices were not annotated by radiologists. The lack of annotations is a major bottleneck of improvement of the performance of detection. In the case of Fig. 3d, the GGO is not annotated by radiologists in the right lung and treated as a false positive. Therefore, detection is affected by incomplete annotations. Shin et al. converted the grayscale HRCT images to color images, where each channel indicates a grayscale image with a particular contrast. This type of forced

Table 3. Comparison of the proposed framework with competing technique

\begin{tabular}{l|c|c}
\hline ILD patterns & $\begin{array}{c}\text { F-score } \\
\text { of Shin et al. }\end{array}$ & $\begin{array}{c}\text { F-score of the } \\
\text { proposed method }\end{array}$ \\
\hline Consolidation & 0.16 & 0.70 \\
Emphysema & 0.35 & 0.55 \\
Fibrosis & 0.75 & 0.68 \\
GGO & 0.56 & 0.58 \\
Healthy & 0.22 & 0.70 \\
Micronodule & 0.71 & 0.86 \\
\hline
\end{tabular}

conversion may limit the proper transfer of contextual information between the two image types. We have avoided the conversion of grayscale images to RGB. Moreover, the method of Shin et al. is able to find the absence or presence of pathology in an HRCT slice. Their method is unable to tell the location of ILD patterns within the slice. The proposed method has true potential to detect the ILD patterns in a lung HRCT slice effectively.

\subsection{Qualitative Results}

Figure 3 shows the qualitative results of the proposed framework for the detection of ILD patterns using without lung field segmentation. In this figure, the ground truth and output bounding box are represented by red and yellow colors, respectively. The results show the effectiveness of the proposed framework in the detection of ILD patterns (Figs. 3a-3f). Figure $3 b$ having two ROIs of GGO, but only one ROI was detected by the proposed framework, marked yellow.

\section{CONCLUSIONS}

In this paper, automated detection of different ILD patterns in lung HRCT images is performed using faster R-CNN based detector network. The proposed framework is able to detect ILD patterns in lung HRCT slices using only five inception blocks in GoogLeNet. The proposed framework performance is competent with research work by Shin et al. The proposed method avoids the hassles of lung field segmentation. The result shows that the conversion of grayscale biomedical images to color images is not manda- 
tory for the detector network. This detector framework could be used in the screening of ILD.

\section{ACKNOWLEDGMENTS}

The authors are grateful to EKO DIAGNOSTICS Kolkata and Kolkata Medical College and for providing useful suggestions for this research work.

\section{FUNDING}

The research was support by grant PhDMLA/4(29)/2015-16 dated April 29, 2016 from Visvesvaraya PhD scheme of DeitY (Department of Electronics and Information Technology), Govt. of India.

\section{COMPLIANCE WITH ETHICAL STANDARDS}

This article is a completely original work of its authors; it has not been published before and will not be sent to other publications until the PRIA Editorial Board decides not to accept it for publication.

\section{Conflict of Interest}

The process of writing and the content of the article does not give grounds for raising the issue of a conflict of interest.

\section{REFERENCES}

1. M. Anthimopoulos, S. Christodoulidis, L. Ebner, T. Geiser, A. Christe, and S. Mougiakakou, "Semantic segmentation of pathological lung tissue with dilated fully convolutional networks," IEEE J. Biomed. Health Inf. 23, 714-722 (2018).

https://doi.org/10.1109/JBHI.2018.2818620

2. M. Anthimopoulos, S. Christodoulidis, L. Ebner, A. Christe, and S. Mougiakakou, "Lung pattern classification for interstitial lung diseases using a deep convolutional neural network," IEEE Trans. Med. Imaging 35, 1207-1216 (2016).

https://doi.org/10.1109/TMI.2016.2535865

3. U. Bagci, J. Yao, A. Wu, J. Caban, T. N. Palmore, A. F. Suffredini, O. Aras, and D. J. Mollura, "Automatic detection and quantification of tree-in-bud (TIB) opacities from CT scans," IEEE Trans. Biomed. Eng. 59, 1620-1632 (2012). https://doi.org/10.1109/TBME.2012.2190984

4. G. Chassagnon, M. Vakalopoulou, E. Battistella, S. Christodoulidis, T. N. Hoang-Thi, S. Dangeard, E. Deutsch, et al., "AI-driven quantification, staging, and outcome prediction of COVID-19 pneumonia,” Med. Image Anal. 67, 101860 (2021). https://doi.org/10.1016/j.media.2020.101860

5. A. Depeursinge, D. V. Ville, A. Platon, A. Geissbuhler, P. A. Poletti, and H. Muller, "Near-affine-invariant texture learning for lung tissue analysis using isotropic wavelet frames," IEEE Trans. Inf. Technol. Biomed. 16, 665-675 (2012).

https://doi.org/10.1109/TITB.2012.2198829
6. S. Christian, W. Liu, Y. Jia, P. Sermanet, S. Reed, D. Anguelov, D. Erhan, V. Vanhoucke, and A. Rabinovich, "Going deeper with convolutions," in Proc. IEEE Conf. on Computer Vision and Pattern Recognition, Boston, Mass., 2015 (IEEE, 2015).

https://doi.org/10.1109/CVPR.2015.7298594

7. A. Depeursinge, A. Foncubierta-Rodriguez, D. V. Ville, and H. Müller, "Lung texture classification using locally-oriented Riesz components," in Medical Image Computing and Computer-Assisted Intervention - MICCAI 2011, Ed. by G. Fichtinger, A. Martel, and T. Peters, Lecture Notes in Computer Sciences, vol. 6893 (Springer, Berlin, 2011), pp. 231-238. https://doi.org/10.1007/978-3-642-23626-6_29

8. A. Depeursinge, A. Vargas, A. Platon, A. Geissbuhler, P. A. Poletti, and H. Müller, "Building a reference multimedia database for interstitial lung diseases," Comput. Med. Imaging Graphics 36, 227-238 (2012). https://doi.org/10.1016/j.compmedimag.2011.07.003

9. A. El-Baz, A. Soliman, P. McClure, G. Gimel'farb, M. A. El-Ghar, and R. Falk, "Early assessment of malignant lung nodules based on the spatial analysisof detected lung nodules," in 9th IEEE Int. Symp. on Biomedical Imaging, Barcelona, 2012 (IEEE, 2012), pp. 1463-1466. https://doi.org/10.1109/ISBI.2012.6235847

10. M. J. Gangeh, L. Sørensen, S. B. Shaker, M. S. Kamel, M. D. Bruijne, and M. Loog, "A texton-based approach for the classification of lung parenchyma in CT images," in Medical Image Computing and ComputerAssisted Intervention - MICCAI 2010, Ed. by T. Jiang, N. Navab, J. P. W. Pluim, and M. A. Viergever, Lecture Notes in Computer Sciences, vol. 6363 (Springer, Berlin, 2010), pp. 595-602.

https://doi.org/10.1007/978-3-642-15711-0_74

11. M. Gao, U. Bagci, L. Lu, A. Wu, M. Buty, H. C. Shin, H. Roth, G. Z. Papadakis, A. Depeursinge, R. M. Summers, Z. Xu, and D. J. Mollura, "Holistic classification of CT attenuation patterns for interstitial lung diseases via deep convolutional neural networks," Comput. Methods Biomech. Biomed. Eng.: Imaging Visualization 6, 1-6 (2016). https://doi.org/10.1080/21681163.2015.1124249

12. C. Goutte, and E. Gaussier, "A probabilistic interpretation of precision, recall and $F$-score, with implication for evaluation," in Advances in Information Retrieval, Ed. by D. E. Losada and J. M. Fernández-Luna, Lecture Notes in Computer Sciences, vol. 3408 (Springer, Berlin, 2005), pp. 345-359. https://doi.org/10.1007/978-3-540-31865-1_25

13. K. He, X. Zhang, S. Ren, and J. Sun, "Deep residual learning for image recognition," in Proc. IEEE Conf. on Computer Vision and Pattern Recognition, 2016 (IEEE, 2016), pp. 770-778.

14. S. Huang, F. Lee, R. Miao, Q. Si, C. Lu, and Q. Chen, "A deep convolutional neural network architecture for interstitial lung disease pattern classification," Med. Biol. Eng. Comput. 58, 725-737 (2020). https://doi.org/10.1007/s11517-019-02111-w

15. C. Jacobs, C. I. Sánchez, S. C. Saur, T. Twellmann, P. A. de Jong, and B. van Ginneken, "Computer-aided detection of ground glass nodules in thoracic CT images using shape, intensity and context features," in Med- 
ical Image Computing and Computer-Assisted Intervention - MICCAI 2011, Ed. by G. Fichtinger, A. Martel, and T. Peters, Lecture Notes in Computer Sciences, vol. 6893 (Springer, Berlin, 2011), pp. 207-214. https://doi.org/10.1007/978-3-642-23626-6_26

16. B. G. Jankharia and B. A. Angirish, "Computer-aided quantitative analysis in interstitial lung diseases-A pictorial review using CALIPER," Lung India 38, 161167 (2021). https://doi.org/10.4103/lungindia.lungindia_244_20

17. D. P. Kingma and J. Ba, "Adam: A method for stochastic optimization." arXiv:1412.6980 [cs.LG]

18. P. D. Korfiatis, A. N. Karahaliou, A. D. Kazantzi, C. Kalogeropoulou, and L. I. Costaridou, "Texturebased identification and characterization of interstitial pneumonia patterns in lung multidetector CT," IEEE Trans. Inf. Technol. Biomed. 14, 675-680 (2010). https://doi.org/10.1109/TITB.2009.2036166

19. G. M. Mallow, Z. K. Siyaji, F. Galbusera, A. A. Espinoza-Orías, M. Giers, H. Lundberg, C. Ames, J. Karppinen, P. K. Louie, F. M. Phillips, R. Pourzal, J. Schwab, D. M. Sciubba, J. C. Wang, H.-J. Wilke, F. M. K. Williams, S. A. Mohiuddin, M. C. Makhni, N. A. Shepard, H. S. An, and D. Samartzis, "Intelligence-based spine care model: A new era of research and clinical decision-making," Glob. Spine J. 11, 135145 (2021). https://doi.org/10.1177/2192568220973984

20. P. Marentakis, P. Karaiskos, V. Kouloulias, N. Kelekis, S. Argentos, N. Oikonomopoulos, and C. Loukas, "Lung cancer histology classification from CT images based on radiomics and deep learning models," Med. Biol. Eng. Comput. 59, 215-226 (2021). https://doi.org/10.1007/s11517-020-02302-w

21. S. C. Park, J. Tan, X. Wang, D. Lederman, J. K. Leader, S. H. Kim, and B. Zheng, "Computer-aided detection of early interstitial lung diseases using low-dose CT images," Phys. Med. Biol. 56, 1139-1153 (2011). https://doi.org/10.1088/0031-9155/56/4/016

22. D. Bermejo-Peláez, S. Y. Ash, G. R. Washko, R. S. J. Estépar, and M. J. Ledesma-Carbayo, "Classification of interstitial lung abnormality patterns with an ensemble of deep convolutional neural networks," Sci. Rep. 10, 338 (2020). https://doi.org/10.1038/s41598-019-56989-5

23. D. R. Sarvamangala and R. V. Kulkarni, "Convolutional neural networks in medical image understanding: a survey," Evol. Intell. (2021). https://doi.org/10.1007/s12065-020-00540-3

24. H. C. Shin, H. R. Roth, M. Gao, L. Lu, Z. Xu, I. Nogues, J. Yao, D. Mollura, and R. M. Summers, "Deep convolutional neural networks for computeraided detection: CNN architectures, dataset characteristics and transfer learning," IEEE Trans. Med. Imaging 35, 1285-1298 (2016).

https://doi.org/10.1109/TMI.2016.2528162

25. K. Simonyan and A. Zisserman, "Very deep convolutional networks for large-scale image recognition." arXiv: 1409.1556 [cs.CV]

26. Y. Song, W. Cai, Y. Zhou, and D. D. Feng, "Feature-based image patch approximation for lung tissue classification," IEEE Trans. Med. Imaging 32, 797-808 (2013). https://doi.org/10.1109/TMI.2013.2241448
27. Y. Song, W. Cai, H. Huang, Y. Zhou, D. D. Feng, Y. Wang, M. J Fulham, and M. Chen, "Large margin local estimate with applications to medical image classification," IEEE Trans. Med. Imaging 34, 1362-1377 (2015).

https://doi.org/10.1109/TMI.2015.2393954

28. Y. Song, W. Cai, J. Kim, and D. D. Feng, "A multistage discriminative model for tumor and lymph node detection in thoracic images," IEEE Trans. Med. Imaging 31, 1061-1075 (2012).

https://doi.org/10.1109/TMI.2012.2185057

29. Y. Song, W. Cai, S. Eberl, M. J. Fulham, and D. Feng, "Discriminative pathological context detection in thoracic images based on multi-level inference," in Int. Conf. on Medical Image Computing and Computer-Assisted Intervention - MICCAI 2011, Ed. by G. Fichtinger, A. Martel, and T. Peters, Lecture Notes in Computer Science, vol. 6893 (Springer, Berlin, 2011), pp. 191-198. https://doi.org/10.1007/978-3-642-23626-6_24

30. Y. Wang, L. Zhou, M. Wang, C. Shao, L. Shi, S. Yang, Z. Zhang, M. Feng, F. Shan, and L. Liu, "Combination of generative adversarial network and convolutional neural network for automatic subcentimeter pulmonary adenocarcinoma classification," Quant. Imaging Med. Surg. 10, 1249 (2020).

https://doi.org/10.21037/qims-19-982

31. Y. Wang, Y. Zhang, Y. Liu, J. Tian, C. Zhong, Z. Shi, Y. Zhang, and Z. He, "Does non-COVID-19 lung lesion help? investigating transferability in COVID-19 CT image segmentation," Comput. Methods Programs Biomed. 202, 106004 (2021). https://doi.org/10.1016/j.cmpb.2021.106004

32. W. R. Webb, N. L. Muller, and D. P. Naidich, HighResolution CT of the Lung, (Lippincott Williams \& Wilkins, 2014).

33. J. Wright, Y. Ma, J. Mairal, G. Sapiro, T.S. Huang, and S. Yan, "Sparse representation for computer vision and pattern recognition," Proc. IEEE 98, 1031-1044 (2010). https://doi.org/10.1109/JPROC.2010.2044470

34. R. Xu, Y. Hirano, R. Tachibana, and S. Kido, "Classification of diffuse lung disease patterns on high-resolution computed tomography by a bag of words approach," Int. Conf. on Medical Image Computing and Computer-Assisted Intervention - MICCAI 2011, Ed. by G. Fichtinger, A. Martel, and T. Peters, Lecture Notes in Computer Science, vol. 6893 (Springer, Berlin, 2011), pp. 183-190. https://doi.org/10.1007/978-3-642-23626-6_23

35. J. Yao, A. Dwyer, R. M. Summers, and D. J. Mollura, "Computer aided diagnosis of pulmonary infections using texture analysis and support vector machine classification," Acad. Radiol. 18, 306-314 (2011). https://doi.org/10.1016/j.acra.2010.11.013 


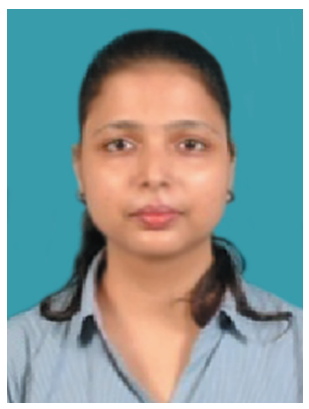

Sunita Agarwala received MTech degree in Information Technology from West Bengal University of Technology, Kolkata, India in 2013. She has completed her PhD degree from National Institute of Technology Durgapur, Durgapur, India. Her research interest includes biomedical image analysis, artificial intelligence, machine learning, and deep learning.

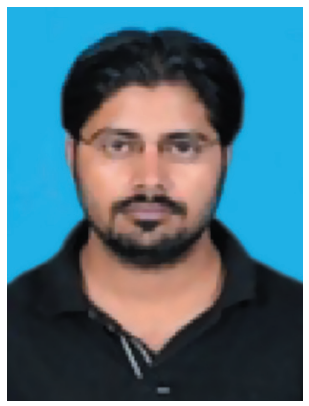

Abhishek Kumar received MTech degree in Artificial Intelligence from University of Hyderabad, Hyderabad, India in 2013. His research interests includes medical image analysis, artificial intelligence, machine learning, and deep learning.

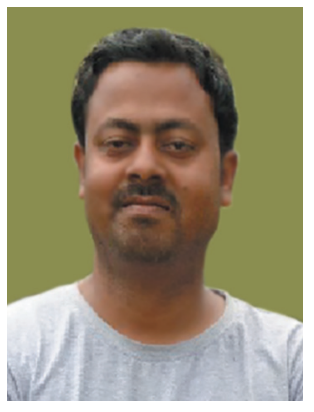

Ashis Kumar Dhara is presently serving as Assistant Professor in Department of Electrical Engineering of National Institute of Technology Durgapur. He received $\mathrm{PhD}$ from Indian Institute of Technology Kharagpur, Kharagpur, India, on medical imaging analysis. His research interest include image processing, deep learning, artificial intelligence, and machine learning.

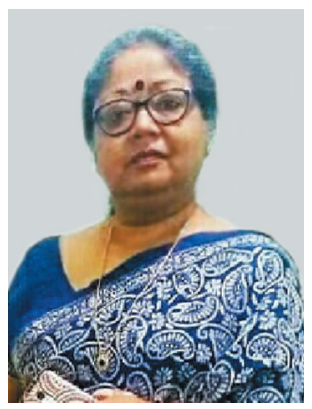

Sumitra Basu Thakur received MBBS from Medical College, Kolkata, India in 1981 and MD in Pulmonary Medicine in 1992 from Medical College, Kolkata. She is presently a Professor and Head of the Department of Chest and Respiratory Care Medicine, Medical College Kolkata, Kolkata, India.

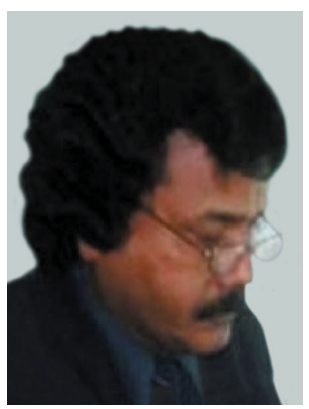

Anup Sadhu received DMRD from the University of Calcutta, Calcutta, India in 1988. He is now Chief Consultant and In-Charge, EKO Diagnostics, Medical College Campus 88, College Street Kolkata-700073. His research interest includes paediatric radiology and non-vascular interventions.

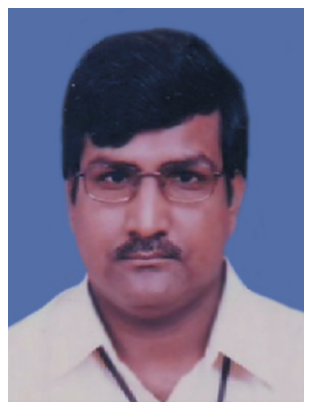

Debashis Nandi received $\mathrm{PhD}$ degree from Indian Institute of Technology Kharagpur, Kharagpur, India, on Medical Imaging Technology. $\mathrm{He}$ is now a Professor in the Department of Computer Science and Engineering, National Institute of Technology Durgapur, Durgapur, India. His research interest includes signal, image and video processing, communication security medical image analysis, and application of machine learning. 